\title{
Effect of Deformation Delayed of the Concrete on the Seismic Response of Shear Walls Strengthened by Composites with a Sinusoidal Distribution of Fibers
}

\section{Effet des Déformations Différées du Béton sur la Réponse Sismique des Murs de Contreventement Renforcés par des Composites ayant une Répartition Sinusoïdale des Fibres}

\author{
R. YEGHNEM ${ }^{1,2}$, L. BOULEFRAKH ${ }^{2}$, S.A. MEFTAH ${ }^{2}$, A. TOUNSI $^{2}$ et E.A. ADDA BEDIA ${ }^{2}$ \\ 1. Université Dr. Tahar MOULAY, Département de Génie Civil et Hydraulique, Saida, Algérie \\ 2. Laboratoire des Matériaux et Hydrologie, Université Djillali LIABES, Sidi Bel Abbés, Algérie
}

\begin{abstract}
The present paper deals with the effect of deformation delayed creep and shrinkage of the concrete on the seismic response of shear walls reinforced concrete strengthened with composite materials having a sinusoidal distribution of fibers. The lateral stiffness of shear walls RC, adhesives and composite material taking into account the phenomenon of creep and shrinkage of concrete described by the Eurocode 2, and widthwise varying fiber volume fraction of the composite plate has been determined by a finite element model. Large earthquakes recorded in Algeria (El-Asnam and Boumerdes) have been tested to demonstrate the accuracy of the proposed method. Numerical results obtained are discussed and the factors influencing the seismic response of reinforced concrete shear walls strengthened taking account the effects of the delay mechanism creep and shrinkage of concrete are highlighted. Prospects are being studied
\end{abstract}

Résumé. Le présent article traite l'effet des déformations différées fluage-retrait du béton sur la réponse sismique des murs de contreventement en béton armé renforcés par des matériaux composites ayant une répartition sinusoïdale des fibres. La rigidité latérale de l'assemblage collé : mur en B.A., adhésif et matériau composite, tenant compte du phénomène du fluage et retrait du béton décrit par l'Eurocode 2 et de la variation de la fraction volumique des fibres selon la largeur de la plaque composite, a été déterminée par un modèle en éléments finis. Des séismes importants enregistrés en Algérie (El-Asnam et Boumerdés) ont été testés afin de démontrer l'efficacité de la méthode proposée. Des matériaux composites à base de fibres de carbone associées à une matrice d'époxyde ont été utilisés.

Les résultats numériques obtenus sont discutés et les facteurs essentiels influençant la réponse sismique des murs de contreventement en béton armé renforcés, tenant compte des 
effets du mécanisme différé fluage-retrait du béton, sont mis en relief. Des perspectives sont en cours d'étude.

\section{Introduction}

Dans les édifices élancés en béton armé, les murs de contreventement en béton armé peuvent être une forme de construction recherchée compte tenu de leur comportement satisfaisant face aux forces de séisme et/ou de vent. Leur grande rigidité en plan contribue à contrôler les déflections et à minimiser les déplacements latéraux. Cependant, ces systèmes structuraux sont nécessaires pour résister sans s'effondrer et sans subir de gros dégâts. Pour atteindre ces objectifs, la structure a besoin d'une ductilité élevée, d'une rigidité suffisante en cisaillement pour une meilleure dissipation de l'énergie. Ces exigences sont souvent incapables de satisfaire les concepteurs en génie civil. Cela ressort clairement de ce qui s'est passé durant les récents séismes en Iran, Turquie, Espagne, Japon, USA et Algérie.

Cependant, plusieurs techniques ont été développées afin d'obtenir une rigidité suffisante des murs de contreventement en béton armé, parmi elles citons les matériaux composites à base de fibres présentant plusieurs avantages : résistances mécaniques élevées, légèreté et facilité de mise en œuvre.

L'utilisation de ces polymères à base de fibres collés en surface pour le renforcement des murs en béton armé est aujourd'hui une technique éprouvée. Leurs applications à travers le monde, à la fois nombreuses et diversifiées, connaissent, depuis leur introduction dans le monde de la construction, un succès remarquable.

Par conséquent, peu de recherches ont été menées sur l'étude du comportement des murs de contreventement renforcés [1...3]. Des études approfondies [4,5] ont montré que les stratifiés de PRFC (polymère renforcé à base de fibres de carbone) collées à l'extérieur sont particulièrement adaptés pour améliorer le comportement à court terme des poutres et dalles en béton armé.
Dans ce papier, une attention est accordée à l'influence des effets rhéologiques du béton, fluage et retrait, sur la rigidité latérale des murs de contreventement renforcés par des plaques en PRFC, ayant une distribution sinusoïdale des fibres, sous deux séismes importants enregistrés en Algérie (El-Asnam et Boumerdés).

\section{Analyse des murs de contreventement par éléments finis}

De nos jours, la méthode des éléments finis est devenue un outil efficace pour l'analyse des structures en béton armé. Dans cet article, deux éléments existants, nommés respectivement : l'élément CHEUNG et l'élément $\operatorname{KWAN}[6,7]$ ont été utilisés. La combinaison de ces deux éléments a mené à un modèle satisfaisant certains critères: l'élément de KWAN simplifié (8 degrés de liberté).

\section{Théories et solutions}

\subsection{Effet du fluage et du retrait sur la déformation élastique des murs en B.A.}

En supposant que le fluage et retrait sont indépendants, la déformation du retrait $\varepsilon_{b}^{F}(y)$ est donnée par [8] :

$\varepsilon_{b}^{F}(y)=\varepsilon_{s b}\left(t-t_{b c}\right)=\alpha T_{b}$

Où : $\alpha, T_{b}$ et $\varepsilon_{s b}\left(t-t_{b c}\right)$ sont respectivement : le coefficient linéaire de dilatation thermique, distribution de température et la déformation du retrait. A partir de (1), il est clair que la déformation du retrait $\varepsilon_{b}^{F}(y)$ est liée à la dilatation thermique. 
La déformation $\varepsilon_{b}(y)$ du mur voile en B.A. peut être écrite sous la forme :

$\varepsilon_{b}(y)=\varepsilon(y)+\alpha T_{b}$

Où $\varepsilon(y)$ est la déformation verticale du mur voile en B.A. définie par :

$\varepsilon(y)=\frac{d v}{d y}$

Remarquons dans les équations suivantes que : $E_{b}=E_{b}(t)$ et $G_{b}=G_{b}(t)$ sont respectivement les modules d'élasticité et de cisaillement dépendant du temps, définis par [9]:

$$
\begin{aligned}
& E_{b}(t)=\frac{E_{b 1}}{1+\chi \varphi\left(t, t_{b}\right)} \\
& G_{b}(t)=\frac{E_{b}(t)}{2(1+v)}
\end{aligned}
$$

\subsection{Fraction volumique des fibres $V_{f}$}

L'expression de la fraction volumique des fibres de matériaux composites prenant compte la variation des propriétés matérielles le long de la largeur de la plaque composite utilisée s'écrit de la façon [10]:

$$
V_{f}=V_{f a v}+A \cdot \cos \left(\frac{2 \pi x}{b}\right)
$$

Où : $V_{f a v}=0.5$ est la valeur moyenne de la fraction volumique des fibres, $A$ l'amplitude sinusoïdale allant de -0.4 à 0.4 avec un pas de 0.1 .

\subsection{Modules élastiques du composite}

Les modules d'élasticité : longitudinale $E_{y}$, transversale $G_{x y}$ et le coefficient de poisson $v_{x y}$ du matériau composite peuvent être donnés, en fonction des propriétés des fibres et de la matrice, en respectant la loi de mélange :

$$
\begin{aligned}
& E_{y}=E_{m} \cdot \frac{E_{m}\left(1-\sqrt{V_{f}}\right)+E_{f} \cdot \sqrt{V_{f}}}{E_{m} \cdot\left[1-\sqrt{V_{f}} \cdot\left(1-\sqrt{V_{f}}\right)\right]+E_{f} \cdot \sqrt{V_{f}}\left(1-\sqrt{V_{f}}\right)} \\
& G_{x y}=G_{m} \cdot \frac{G_{m} \sqrt{V_{f}}\left(1-\sqrt{V_{f}}\right)+G_{f} \cdot\left[1-\sqrt{V_{f}}\left(1-\sqrt{V_{f}}\right)\right]}{G_{m} \cdot \sqrt{V_{f}}+G_{f} \cdot\left(1-\sqrt{V_{f}}\right)} \\
& v_{x y}=v_{m}\left(1-\sqrt{V_{f}}\right)+v_{f} \sqrt{V_{f}}
\end{aligned}
$$

\subsection{Matrice de rigidité du mur de contreventement renforcé}

Le calcul de la matrice de rigidité du mur en béton armé renforcé par des plaques en matériau composite (voir fig.1), nécéssite la détermination de l'énergie de déformation de l'élément, due aux effets de flexion et de cisaillement, définie par:
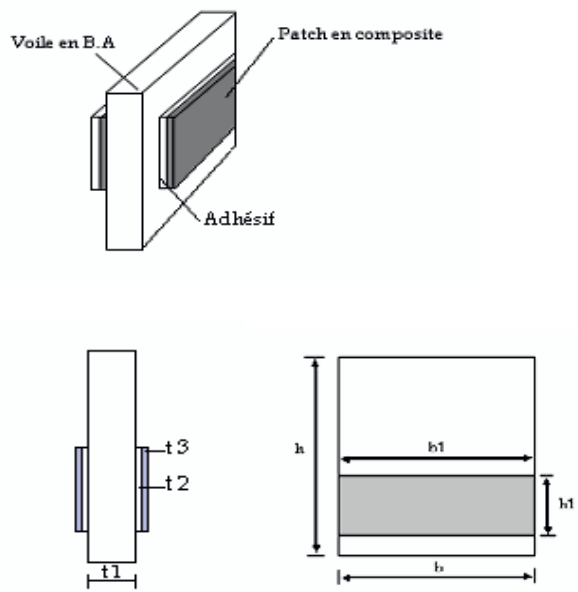

Fig. 1. Perspective d'emplacement des plaques composites

(1) Emplacement des plaques, (2) Vue de face et (3) Vue latérale

$U^{e}=U_{F}^{e}+U_{c}^{e}$

Où $U_{f}^{e}$ et $U_{c}^{e}$ sont respectivement les énergies de déformation dues à la flexion et au cisaillement, écrites en fonction des déformations du mur de contreventement.

Considérons l'énergie de déformation due à l'effet de flexion, notée $U_{f}^{e}$ : 


$$
U_{f}^{e}=\frac{1}{2} \sum_{i=1}^{3} \int_{v o l} E_{y}^{(i)}\left(\varepsilon_{y}\right)^{2} d \operatorname{vol}_{(i)}
$$

L'expression de l'énergie de déformation due au cisaillement peut être écrite comme suit :

$U_{c}^{e}=\frac{1}{2} \sum_{i=1}^{3} \int_{v o l} G_{x y}\left(\gamma_{y}\right)^{2} d \operatorname{vol}_{(i)}$

Pour l'élément mur, l'énergie de déformation s'écrit de la façon:

$$
U^{e}=\frac{1}{2} d_{e}^{t} \cdot K_{w} \cdot d_{e}
$$

Où le vecteur de déplacement nodal $d_{g}$ est donné par:

$d_{e}=\left\{u_{1}, \omega_{1}, v_{1}, v_{2}, u_{2}, \omega_{2}, v_{3}, v_{4}\right\}$

La matrice de rigidité $K_{w}$ est déterminée par une formulation en éléments finis standards.

\subsection{Résolution de l'équation du mouvement}

L'équation générale de mouvement peut être écrite sous la forme :

$$
[M] .\{\ddot{U}(t)\}+[C] \cdot\{\dot{U}(t)\}+[K] .\{U(t)\}=\{P(t)\}
$$

Où: $[\mathrm{M}],[\mathrm{C}]$ et $[\mathrm{K}]$ désignent respectivement les matrices masse, d'amortissement et de rigidité.

$\{\ddot{U}(t)\},\{\dot{U}(t)\}$ et $\{U(t)\}$ : respectivement les vecteurs d'accélération, de vitesse et de déplacement.

$\{P(t)\}$ : Vecteur des forces extérieures.

La matrice d'amortissement est calculée par la méthode de Rayleigh par:

$$
[C]=\alpha_{1}[M]+\alpha_{2}[K]
$$

Avec:

$$
\alpha_{1}=\xi \frac{2 \lambda_{j} \lambda_{p}}{\lambda_{j}+\lambda_{p}} \text { et } \alpha_{2}=\xi \frac{2}{\lambda_{j}+\lambda_{p}}
$$

$\xi$ : facteur d'amortissement du système $(5 \%)$

$\lambda_{j}$ et $\lambda_{p}$ : fréquences propres déterminées par la résolution de l'équation du système non amorti :

$\left|[K]-\lambda^{2}[M]\right|=0$

Par l'utilisation de la méthode d'intégration pas à pas de Newmark [11], l'équation du movement a été résolue. Les paramètres $\beta$ et $\gamma$ valent respectivement $1 / 4$ et $1 / 2$.

\section{4 Étude numérique}

De nombreux exemples sont présentés pour valider les résultats de la présente méthode. Ces résultats seront comparés à ceux du modèle de référence (modèle non renforcé). Les paramètres géométriques de la structure contreventée et mécaniques des matériaux utilisés sont donnés dans les tableaux 1et 2. Les épaisseurs, de l'adhésif et de la plaque, sont respectivement 0.002 et $0.006 \mathrm{~m}$.

Tableau 1. Dimensions de la structure étudiée

\begin{tabular}{|c|c|c|c|c|}
\hline \multirow{2}{*}{$\begin{array}{c}\text { Structur } \\
\mathrm{e}\end{array}$} & \multicolumn{4}{|c|}{ Dimensions } \\
\cline { 2 - 5 } & $\begin{array}{c}\text { Hauteur } \\
\text { totale } \mathrm{H}\end{array}$ & $\begin{array}{c}\text { Hauteur } \\
\text { d'étage }\end{array}$ & $\begin{array}{c}\text { Largeur } \\
\text { du mur }\end{array}$ & $\begin{array}{c}\text { Epaisseu } \\
\mathrm{r} \mathrm{du} \text { mur }\end{array}$ \\
\hline 25 étages & $75 \mathrm{~m}$ & $3 \mathrm{~m}$ & $12 \mathrm{~m}$ & $0.25 \mathrm{~m}$ \\
\hline
\end{tabular}

Tableau 2. Paramètres mécaniques des matériaux utilisés

\begin{tabular}{|c|c|c|}
\hline Matériaux & $\begin{array}{c}\text { Module de Young } \\
\mathrm{E}(\mathrm{GPa})\end{array}$ & $\begin{array}{c}\text { Coefficient de } \\
\text { Poisson } v\end{array}$ \\
\hline Béton & 30 & 0.18 \\
\hline Adhésif & 3 & 0.35 \\
\hline Epoxyde & 3,445 & 0.35 \\
\hline Carbone & 140 & 0.22 \\
\hline
\end{tabular}




\subsection{Accélérogrammes sismiques}

Pour mener l'analyse dynamique, deux séismes ont été appliqués: El-Asnam et Boumerdés. Leurs accélérogrammes et caractéristiques sont données dans le tableau 3 et la figure 2 .

Tableau 3. Caractéristiques des séismes choisis

\begin{tabular}{|c|c|c|c|c|}
\hline Séisme & Localisation & Date & $\begin{array}{c}\text { Accélération } \\
\text { max du } \\
\text { sol.PGA }\end{array}$ & $\begin{array}{c}\text { Magnitud } \\
\mathrm{e}\end{array}$ \\
\hline El-Asnam & $\begin{array}{c}\text { El-Asnam } \\
\text { (Chlef) }\end{array}$ & $\begin{array}{c}10- \\
10- \\
198\end{array}$ & $0.049 \mathrm{~g}$ & 7.3 \\
& & 0 & & \\
\hline Boumerdés & Région & $21-$ & $0.35 \mathrm{~g}$ & 6.8 \\
& d'Alger & $05-$ & & \\
& Boumerdés : & 200 & & \\
& Keddara & 3 & & \\
\hline
\end{tabular}

(a)

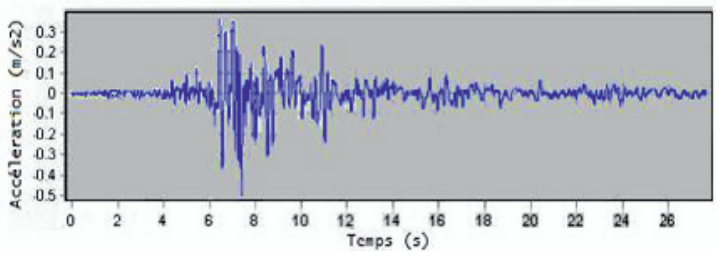

(b)

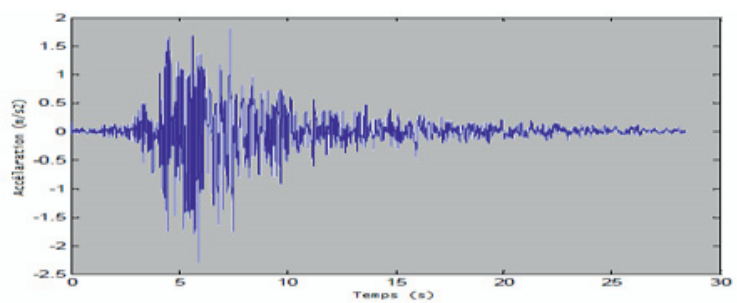

Fig. 2. Accélérogrammes des séismes

(a) El-Asnam et (b) Boumerdés

\subsection{Présentation des résultats}

D'une part, les déplacements latéraux des murs de contreventement renforcés par des PRFC sont comparés à ceux du modèle de référence (non renforcé), sous les deux séismes choisis (El-Asnam et Boumerdés), en tenant compte de la distribution sinusoïdale des fibres de la plaque et l'effet du fluage et retrait du béton (âge du béton $\mathrm{t}=120$ jours).. Les résultats sont portés sur la figure 3 .
D'autre part, les déplacements latéraux des murs de contreventement, renforcés par des plaques en composites à base de fibres de carbone sont étudiés sous le séisme de Boumerdés, en tenant compte de l'effet du fluage et retrait du béton (âge du béton $\mathrm{t}=$ $120 \ldots 5000$ jours). Cet effet est reporté sur la figure 4.

(a)

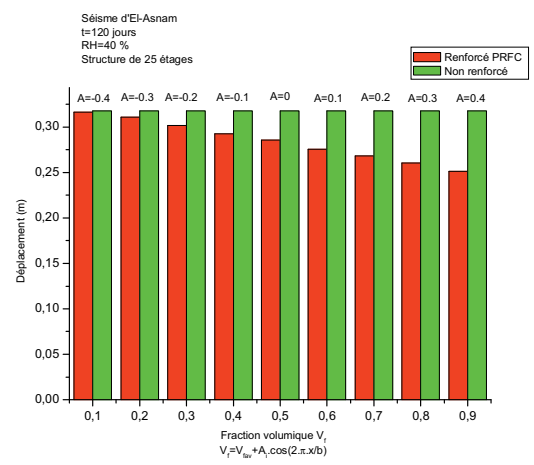

(b)

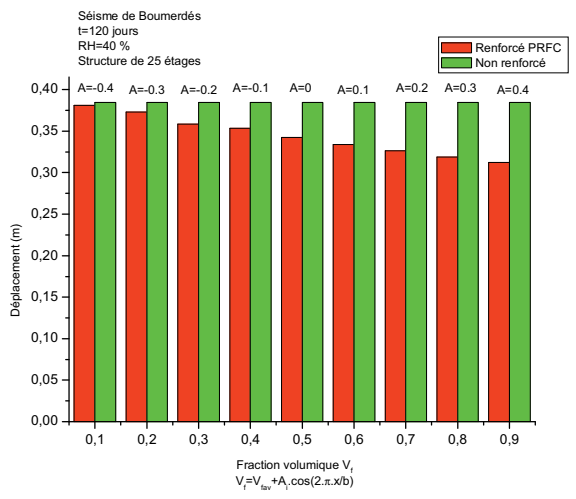

Fig. 3. Effet de la distribution des fibres du composite sur les déplacements sous les deux séismes

(a) El Asnam et (b) Boumerdés 


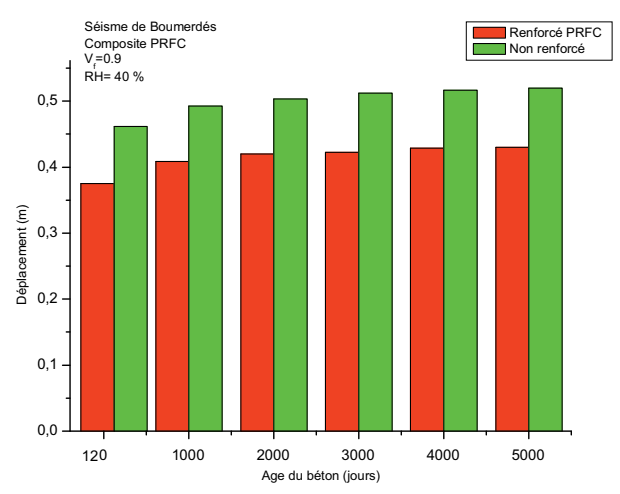

Fig. 4. Effet du fluage et retrait sur les déplacements:

Séisme de Boumerdés

\subsection{Discussion des résultats}

Analysons l'effet de la distribution sinusoïdale des fibres des plaques composites utilisées dans la présente étude pour le renforcement des murs de contreventement sous les séismes testés et d'après les résultats obtenus, nous pouvons constater que si la fraction volumique des fibres avoisine les 0.9, les déplacements latéraux des murs diminuent de $20 \%$ sous le séisme d'El-Asnam et de $18 \%$ pour le séisme de Boumerdés.

La réduction des déplacements est donc proportionnelle à la variation de la fraction des fibres le long de la largeur de la plaque composite. De ce fait, les fibres doivent être concentrées aux bords de la plaque composite où les contraintes sont les plus élevées.

Par l'analyse de l'effet du fluage et retrait du béton sur les déplacements des murs, la réduction des déplacements est notamment proportionnelle à l'âge du béton. De ce fait, pour un jeune âge, les déplacements des murs sont tolérables. Ils deviennent élevés au cours $\mathrm{du}$ temps et se stabilisent à long terme (généralement au delà de 7000 jours).

\section{Conclusion}

Le but de cette contribution est d'analyser l'effet des déformations différées fluage et retrait sur le comportement sismique des murs de contreventement en béton armé renforcés par des plaques en composite ayant une répartition sinusoidale des fibres. Un modèle en éléments finis a été établi afin de déterminer les déplacements latéraux des murs renforcés sous des séismes locaux enregistrés en Algérie (ElAsnam et Boumerdés). Les principales conclusions à retenir sont :

- Les déplacements augmentent au cours du temps sous l'effet du fluage du béton ;

- Le renforcement dépend des caractéristiques mécaniques et géométriques des plaques composites utilisées ;

- L'influence du fluage est constatée au bout des premiers mois de durcissement du béton.

\section{References}

[1] A. Balsamoa, A. Colombob, G. Manfredic, P. Negrod and A. Protac, "Seismic behavior of a fullscale RC frame repaired using CFRP laminates", Engineering Structures, Vol. 27, 769-780, (2005).

[2] S.A. Meftah, A. Tounsi, A. Megueni And E. A. Adda Bedia, "Lateral stiffness and vibration characteristics of RC shear walls with variable fibres spacing", Materials \& Design, Vol. 29, 1955-1964, (2008).

[3] R. Yeghnem, S.A. Meftah, A. Tounsi and E.A. Adda Bedia," Earthquake response of RC coupled shear walls strengthened with thin composite plates", Journal of vibration and control, Vol. 15, 963-985, (2009).

[4] S. Benyoucef, A. Tounsi, S.A. Meftah and E.A. Adda Bedia, "Approximate analysis of the interfacial stress concentrations in FRP - RC hybrid beams, Composite interfaces, Vol. 13,561-571, (2006).

[5] H.S. Shen, Y. Chen and J. Yang, "Bending and vibration characteristics of a strengthened plate under various boundary conditions", Engineering Structures, Vol. 25, 1157-1168, (2003).

[6] Y. K. Cheung and S. Swaddiwudhipong, "Analysis of frame shear wall structures using finite strip elements", In Proceedings of the Institution of Civil Engineers, Part 2, 65, September 517-535. (1978).

[7] A.K.H. Kwan, "Local deformations and rotational degrees of freedom at beam-wall joints", Computer \& Structures, Vol. 48, 615-625, (1993).

[8] EUROCODE 2, Design of concrete structures. Pt 1-1: General Rules and Rules for Buildings. Editorial Group, Brussels (1991).

[9] H. Trost and J. Wolff, "Zur wirklichkeitsnahen ermittlung der beanspruch-ungen in abschnittsweise hergestellten spannbetontragwerken", Der Bauingenieur, 45, 155-169, (1970). (In German)

[10] T. Kubiak, "Dynamic buckling of thin-walled composite plates with varying widthwise material 
properties", Solids \& Structures, Vol. 42, 55555567, (2005).

[11] K.J. Bathe and E.L. Wilson, "Numerical Methods in finite element analysis", Prentice -Hall ISBN 0136271901, 9780136271901, (1976). 